\title{
A new approach to patient indexing
}

\author{
J C W EDWARDS, K THOMPSON, AND D G JAMESON
}

From the Departments of Rheumatology and Computer Services, University College and Middlesex Hospital Medical School, London

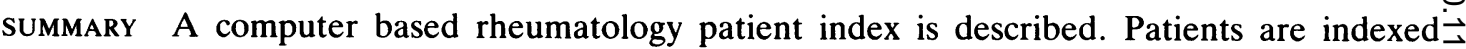
according to essential clinical and laboratory data without reference to Procrustean diagnostic ${ }_{\sigma}^{\omega}$ labels. The stored information can be searched for a wide range of practical and scientific purposes. The software was devised in the windows environment for the Apricot Xen for maximum speed and intelligibility.

The philosophy behind this index has been outlined in a viewpoint article in the Annals of the Rheumatic Diseases. ${ }^{1}$ The basic assumption in designing the index was that diagnostic categories in current use are less useful than the clinical and laboratory evidence of disease processes from which they are derived. This applies equally to clinical practice and scientific enquiry.

The index was set up to establish whether a core of information could be gathered on new outpatient attenders which required no more effort than a traditional 'diagnostic' index, but which could provide a more reliable and versatile database.

\section{Detailed description}

SOFT T A RE

A software package was devised by KT using the computer language ' $C$ ', the windows environment, and Apricot Xen hardware. The software was designed solely for the index. On starting up the index is entered immediately, avoiding time consuming and confusing menu options. The data files created, however, can be used for other purposes if required.

The windows environment is pleasant to the eye, allows handling with a mouse or with the keyboard, and the overlay system allows the user to keep in touch' with general information about the file being created. The software consists of three main program units; for entering data, for searching, and for printing out.

\section{DATA COLLECTION}

Printed sheets carrying an inventory of clinical and

Accepted for publication 16 May 1987.

Correspondence to Dr J C W Edwards, Rheumatology Research Department, Arthur Stanley House, Tottenham St, London W1 1PG. laboratory features and associated alpha-numeric codes are put loose into the case notes of ale new outpatient attenders. (Previously dischargedpatients reattending for new problems are treated as new.) Medical staff ring the chosen codes, and theo sheets are collected together. Once a month the datad are entered on the computer, every sheet having $\overrightarrow{-}$ been checked by a single doctor (JCWE).

PRINTED SHEETS

The printed sheet is reproduced in Fig. 1. Thres inventory is divided into the following sections: $(i)$ identification, (ii) site(s) of rheumatic problem(s) (iii) type of rheumatic problem, (iv) common associations with rheumatic illness and other common disorders, $(v)$ laboratory information, treat $-\overline{3}$ ment, and name of consultant. Items are allocated? codes. The rather cryptic nature of these codeso relates to complex rules designed to prevent wasteful searches, which will not be described in detail. $A \bar{c}$ space is also provided for 'rarities' which may consisto of up to 50 characters. There is no other restriction. on the entry in this category.

Entries follow rules designed for speed. All patients have section (i) filled in by clinic staff. TheO site of rheumatic problem must be entered, unless $\triangle$ there is no rheumatic problem or pain is of 'dubious soft tissue origin (A00). For common problems, $N$ such as shoulder, neck, or lumbar pain no furtheros details are obligatory, but synovitis, cartilage loss $N$ osteophytes, or nerve entrapment can be added if desired. Certain sites are linked to a typical localo lesion, as for elbow epicondylitis and sacroiliitis Where several joints are affected by a particular process (polyarticular) this must be specified in category (iii). Category (iii) provides information ono rheumatic disease according to the type of diseaseo process. 


\section{PATIENT INDEX FORM}

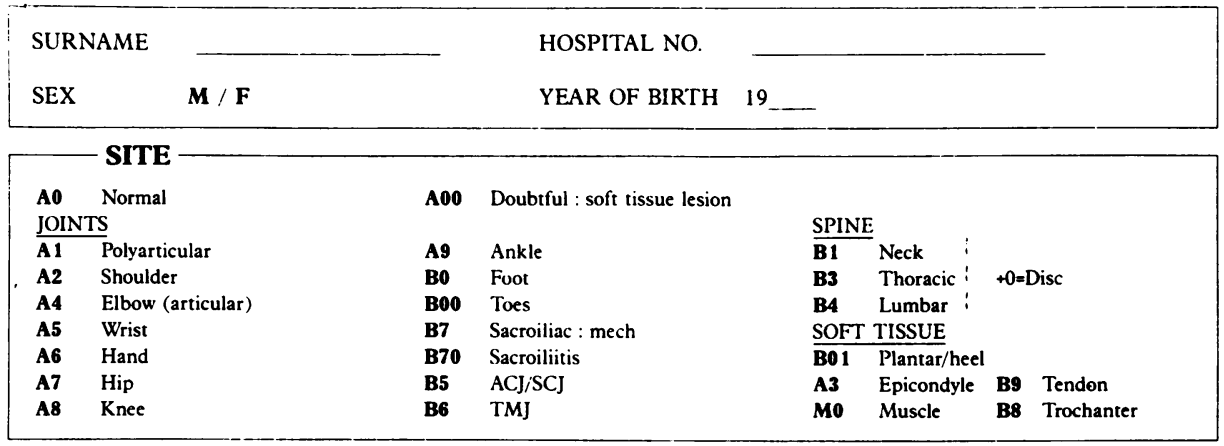

\begin{tabular}{|c|c|c|c|c|c|}
\hline \multicolumn{4}{|c|}{ JOINTS } & \multirow[t]{3}{*}{$\mathbf{K}$} & \multirow[t]{3}{*}{ Childhood onset } \\
\hline $\mathbf{C O}$ & Peripheral joint pain or swelling & $g$ : cause unce & certain & & \\
\hline \multirow[t]{3}{*}{ C1 } & Synovitis, immunological (i.e. no & o other causc & & & \\
\hline & to Non-erosive +1 & Erosive & +2 Nodules & & +3 Palindromic \\
\hline & +4 Post infective & Assymetric & +6 Mutilans & & +7 DIP \\
\hline C2 & Cartilage loss : query cause & C3 & Cartilage loss : cause known & C4 & Osteophytes/Heberden's Nodes \\
\hline G0 & Hyperuricaemia & G1 & Gout & & \\
\hline $\mathbf{x} 1$ & Calcium crystal deposition & II & Joint infection & & \\
\hline \multicolumn{6}{|c|}{ RARITIES : Specify } \\
\hline \multicolumn{2}{|c|}{ SPINE } & \multicolumn{2}{|l|}{ BONE } & \multicolumn{2}{|c|}{ VARIOUS } \\
\hline \multirow[t]{2}{*}{$\overline{\mathbf{C 5}}$} & Spondylitis & $\overline{\mathbf{X O}}$ & Bone disorder & $\mathbf{Y}$ & Trauma : all types \\
\hline & & $\mathbf{X 2}$ & Osteoporosis & 12 & TB \\
\hline \multicolumn{2}{|c|}{ MUSCLE } & $\mathbf{X 3}$ & Osteonecrosis & DO & Amyloid \\
\hline$\overline{\mathbf{M 1}}$ & Polymyalgia & $\mathbf{X 4}$ & Dyspplasia & D2 & Scleroderma/PSS \\
\hline M2 & Myositis & $\mathbf{X 5}$ & Paget's & D3 & Sarcoid \\
\hline & Myopathy & & & D4 & Algodystrophy \\
\hline & & & & C7 & Musculoskeletal tumour \\
\hline & & & & Y1 & Hỵpermobility \\
\hline
\end{tabular}

\begin{tabular}{|c|c|c|c|c|c|}
\hline \multicolumn{2}{|c|}{ SICCA RELATED CLUSTER } & \multicolumn{2}{|c|}{ SPONDYLITIS RELATED CLUSTER } & \multicolumn{2}{|c|}{ MISCELLANEOUS } \\
\hline$\overline{\mathbf{J 0}}$ & Sicca Sx & $\overline{\mathbf{U} 0}$ & Urethritis/Balanitis & $\overline{\mathbf{N 1}}$ & Neuropathy \\
\hline J2 & Lip biopsy +ve & N8 & Conjunctivitis & $\mathbf{N} 10$ & Entrapment \\
\hline U3 & Nephritis & N9 & Uveitis & Po & Psychological component \\
\hline N2 & Cerebritis & E2 & Crohn's/U.C. & vi & Necr. Arteritis \\
\hline S3 & Skin vasculopathy & $\mathbf{E 3}$ & Dysentery & V4 & G.C. Arteritis \\
\hline S4 & Discoid LE & E4 & Mouth ulcers & $\mathbf{w} 1$ & Thyroid \\
\hline S5 & Raynaud's & S1 & Psoriasis & w2 & Diabetes \\
\hline S6 & Photosensitivity/butterfly rash & S2 & Nail dystrophy & $\mathbf{Q}$ & Allergy \\
\hline H2 & Neutropenia & & & & \\
\hline H3 & Lymphopenia & & & & \\
\hline H4 & Thrombocytopenia & & & & \\
\hline V3 & Carditis & & & & \\
\hline L5 & Serositis & & & & \\
\hline $\mathbf{L 3}$ & Pulmonary fibrosis & & & & \\
\hline
\end{tabular}

\section{TESTS / TREATMENT / CONSULTANT}

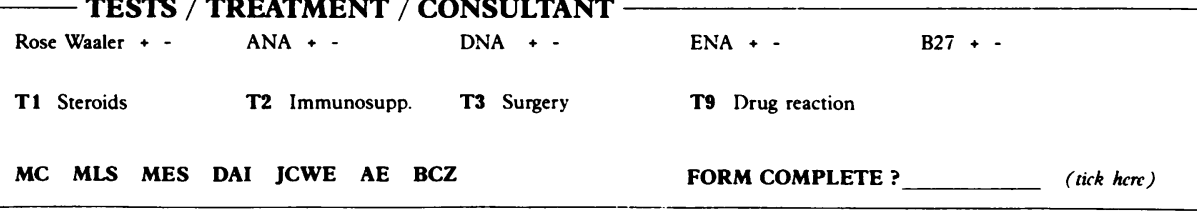

Fig. 1 Inventory of common problems for indexing of patients. Unusual problems are recorded specifically under 'Rarities'. 
Other categories are filled in if appropriate information is available and of interest.

\section{SEARCHING PROCEDURE}

Any single code or combinations of up to three codes can be searched for. Data on patients carrying those codes are printed out in monthly sets.

The index has proved practical. Filling in codes in the clinic takes about 10 seconds. Vetting and entering data on the computer takes an hour each month. Where clinicians had initial difficulty in deciding how to code patients they were encouraged to write problems out manually, and these were discussed before being entered onto the computer.

The index has been running for nine months, and 800 patients have been indexed. There is a lag in indexing in some cases because the doctor can hold the printed sheet back until the second, or occasionally the third, visit so that important laboratory data can be entered. The completion rate is checked against the clinic manager's monthly statistics.

It is possible to establish how many patients with rheumatic problems and psoriasis are seen in a given period. The printout gives a breakdown of how many have spondylitis, synovitis, erosions, etc. A single consultant can see how many patients with shoulder pain he has seen, to assess the impact of introducing a policy of early appointments for such patients, or entry into a treatment study.

In addition to monthly new patient files, an old patient file has been set up. This takes patients of continuing interest, rather than all attenders. A clinician interested in azathioprine can index all his cases on the drug by adding ' $A Z A$ ' to the other codes and search under 'AZA'. A few spurious records may appear with this type of search but are easily weeded out.

As can be seen the information recorded and searched for is highly flexible, but common problems are highlighted by the structured coding $\frac{\square}{\omega}$ system.

\section{Discussion}

The point of departure of this index lies in the idea that clinical and laboratory data should be stored without reference to a diagnostic label. It is far from clear that all rheumatologists have the same concept $\mathrm{c} s$ of the processes represented by terms such as $\overrightarrow{0}$ osteoarthritis and rheumatoid arthritis. If we are to explore the heterogeneity and overlap of different $\vec{\omega}$ elements of these processes then it is inappropriate to store data under such headings.

Users of this index who wish to collect patients by $\overrightarrow{0}$ current diagnostic criteria have no difficulty in doing $\vec{\circ}$ so. Most criteria are recorded in the inventory. A screening index of this sort cannot be expected to ${ }^{\circ}$ provide completely up to date and reliable informa- 0 tion, whatever the coding system used, and all searches must be checked by reference to the notes or by seeing the patients.

Any index is a compromise between speed of use and thoroughness of documentation. The suggested $\stackrel{\mathbb{}}{\stackrel{\mathbb{S}}{ }}$ index is only marginally more complex than pre- $\overrightarrow{0}$ vious indexes used in our combined departments. Because the data have not been reduced to diagnosis, however, it allows rapid searching fo information in a wide range of contexts and combinations. It is not perfect, but we feel it moves in the right direction.

We would like to thank the special trustees of the Middlesex Hospital for financial support for this work.

\section{Reference}

1 Edwards J C W. Diagnostic categories in rheumatology. Ann Rheum Dis 1987; 46: 259-61. 